\title{
Empresas fintech, activos virtuales y la era digital: retos y oportunidades en México
}

\author{
Fintech Companies, Virtual Assets and the Digital Age: Challenges and \\ Opportunities in México
}

Empresas Fintech, ativos virtuais e a era digital: desafios e oportunidades no México

\author{
María de los Ángeles Velázquez Martínez \\ Centro Universitario UAEM Texcoco, México \\ vema640828@hotmail.com \\ https://orcid.org/0000-0001-8149-8012
}

\section{Resumen}

En la era de la $4^{\circ}$ revolución industrial y la disrupción financiera, las tecnologías de la información, la innovación tecnológica, la inteligencia artificial y activos virtuales, entre otros conceptos, han cambiado el modo de vida de la sociedad, el uso de los teléfonos inteligentes para realizar actividades económicas, los medios de pago e inversión que han pasado de los métodos tradicionales de la banca al manejo de los activos virtuales y, el internet como plataforma de uso; sin embargo el tema tecnológico aún resulta desconocido para una gran parte de la población, para otro sector, resulta un camino innovador de inversión y negocio y desafortunadamente para otros, es una fuente para cometer ciberdelitos. Realizando un escrutinio de los diversos conceptos que giran en el entorno de la era digital y analizando la apreciación de diversos especialistas en la materia, se realizó una investigación de tipo descriptiva documental y, utilizando el método deductivo se revisaron las diferentes fuentes de información especializada con el objetivo de profundizar sobre las características de los criptoactivos, activos virtuales y las empresas Fintech, así como los retos y oportunidades que enfrentan en la era de la cuarta revolución industrial y la nueva legislación 
aplicable en materia, así como el riesgo y la vulnerabilidad de éstas empresas a ser utilizadas en la consecución de los ciberdelitos como el fraude y el lavado de activos entre otros, que toman fuerza con la situación actual de la pandemia originada por el COVID-19.

Las empresas Fintech utilizan la innovación digital para ofrecer servicios financieros más eficaces y económicos a la población que no cuenta con la banca tradicional, actualmente teniendo que ajustarse a la nueva normativa regulatoria para su operación, considerando que México ocupa el $2^{\circ}$ lugar en ciberataques, representa un gran reto que todos los usuarios de las tecnologías financieras desarrollen la cultura de implementar ciberseguridad con el objetivo de, prevenir la consecución de los delitos cibernéticos. Asimismo, considerando el contexto actual antes descrito, se crea la oportunidad para el crecimiento y desarrollo económico de las empresas Fintech y los usuarios de las tecnologías financieras.

Palabras clave: activos virtuales, disrupción, tecnologías financieras.

\section{Abstract}

In the 4th industrial revolution and financial disruption stage, the information technologies, technological innovation, artificial intelligence and, virtual assets, among another concepts, have changed the society way of life, the use of smart telephones to carry out economic activities, the payment and investment methods have gone from the traditional methods of banking to the management of virtual assets and, the internet as a platform for use; however, the technological issue is still unknown for a large part of the population, for some sector, it is an innovative way of investment and business, but unfortunately for others, it is the source for cybercrime. Carrying out a search of the various concepts considered in the environment of the digital age and analyzing the appreciation of various specialists in the field, a documentary descriptive research was carried out and, using the deductive method, the different sources of specialized information were reviewed with the aim of go through the characteristics of crypto assets, virtual assets and also Fintech enterprise, as well as the challenges and opportunities they face in the era of the fourth industrial revolution and the new applicable legislation on the matter, as well as the risk and vulnerability of These companies to be used in the pursuit of cybercrimes such as fraud and money wash, among others, that are taking hold with the current situation of the pandemic caused by COVID-19. 


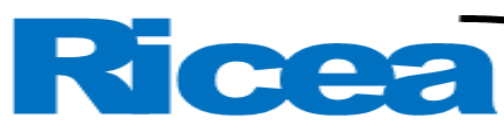

REVISTA IBEROAMERICANA DE CONTADURÍA, ECONOMÍA Y ADMINISTRACIÓN

ISSN= $2007-9907$

Fintech companies use digital innovation to offer more efficient and economic financial services to the population that do not have traditional banking, currently having to adjust to the new regulatory issues for its operation, considering that Mexico ranks 2nd place in cyber attacks, It represents a great challenge for all users of financial technologies to develop the culture of implementing cybersecurity with the aim of preventing the achievement of cybercrime. Also, considering the current context described above, the opportunity for economic growth and development of Fintech companies and users of financial technologies is created.

Keywords: virtual assets, disruption, financial technologies.

\section{Resumo}

In the era of the 4th industrial revolution and financial disruption, information technologies, technological innovation, artificial intelligence and virtual assets, among other concepts, have changed the way of life of society, the use of telephones intelligent to carry out economic activities, the means of payment and investment that have gone from the traditional methods of banking to the management of virtual assets and the internet as a platform for use; However, the technological issue is still unknown for a large part of the population, for another sector, it is an innovative way of investment and business and unfortunately for others, it is a source for committing cybercrimes. Carrying out a scrutiny of the various concepts that revolve in the digital age environment and analyzing the appreciation of various specialists in the field, a descriptive documentary research was carried out and, using the deductive method, the different sources of specialized information were reviewed with The objective of delving into the characteristics of crypto assets, virtual assets and Fintech companies, as well as the challenges and opportunities they face in the era of the fourth industrial revolution and the new applicable legislation on the matter, as well as the risk and vulnerability of These companies to be used in the pursuit of cybercrimes such as fraud and money laundering among others, which gain strength with the current situation of the pandemic caused by COVID-19.

Fintech companies use digital innovation to offer more efficient and economical financial services to the population that does not have traditional banking, currently having to adjust to the new regulatory regulations for their operation, considering that Mexico ranks 2nd in cyberattacks, It represents a great challenge for all users of financial technologies to develop 
a culture of implementing cybersecurity with the aim of preventing the achievement of cybercrimes. Likewise, considering the current context described above, the opportunity is created for the growth and economic development of Fintech companies and users of financial technologies.

Keywords: virtual assets, disruption, financial technologies.

Fecha Recepción: Febrero 2020 Fecha Aceptación: Julio 2020

\section{Introducción}

En la era de la cuarta revolución industrial, conceptos como criptoactivos, monedas virtuales, bitcoin, eros y tecnologías financieras, entre otros, fluyen en la economía digital creando directrices que los usuarios, las empresas de servicios financieros y las autoridades deben tener presentes con el propósito de realizar operaciones financieras rápidas y eficientes para implementar legislación normativa que las regule. De hecho, el uso de las tecnologías financieras ha cambiado el modo de vida de la sociedad, ya que propicia la inclusión financiera y la disminución del efectivo como método de pago. En efecto, los medios de inversión y liquidación han pasado de los métodos tradicionales de la banca al manejo de los activos virtuales, las compras y ventas en línea, y el internet como plataforma de uso. Aun así, el tema tecnológico todavía resulta desconocido para una gran parte de la población, lo que puede servir de fuente para cometer ciberdelitos. Por tal motivo, resulta importante profundizar en ese tipo de conceptos, los cuales se deben entender y operar para que no sean usados en perjuicio nuestro.

En efecto, y como antecedente, se puede decir que México ocupa el segundo lugar en cuanto a la consecución de ciberdelitos, de ahí que hayan sido creadas medidas para regular las operaciones tecnológicas y para prevenir estos delitos financieros. Un ejemplo de ello es la promulgación de la ley para regular a las instituciones de tecnología financiera (ley fintech) publicada en el Diario Oficial de la Federación el 9 de marzo de 2018. Esta, reglamenta las operaciones, autorizaciones, pagos electrónicos, operaciones con activos virtuales, así como su inspección y vigilancia. De este modo se busca proteger a los consumidores y asegurar la confidencialidad de la información de los clientes y usuarios. Sin embargo, hasta antes de la legislación emitida existían alrededor de 515 empresas fintech operando, cifra que disminuyó a 85 luego de la implementación de dicha norma. 


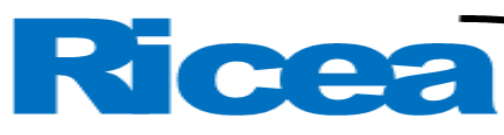

REVISTA IBEROAMERICANA DE CONTADURÍA, ECONOMÍA YADMINISTRACIÓN

En este contexto surgen los siguientes cuestionamientos, los cuales han orientado el desarrollo de la presente indagación: ¿el uso de las tecnologías financieras en las operaciones económicas de compra-venta representan un riesgo o posibilidades de crecimiento para los usuarios? ¿Cuáles son los retos y oportunidades que enfrentan las empresas fintech en la actualidad al operar con activos virtuales? ¿Estas operaciones y empresas pueden ser utilizadas para cometer ciberdelitos o delitos financieros?

\section{Método}

Este trabajo se sustentó en una investigación descriptiva-documental, pues se efectuó un escrutinio a fuentes de información primarias y secundarias disponibles en la Web. Para ello, se empleó el método deductivo y se revisaron los conceptos y teorías sobre las tecnologías financieras, criptoactivos y empresas fintech (qué tipo de operaciones realizan y cuál es la legislación aplicable), cuáles son las características de los activos virtuales que operan en los mercados financieros, qué delitos se pueden perpetrar en el uso de las tecnologías financieras y qué reglamentación y procedimientos se deben seguir para prevenirlos. En síntesis, el objetivo se enfocó en analizar los retos y las oportunidades que enfrentan las fintech y el uso de las tecnologías financieras, así como qué regulación y medidas de prevención deben adoptar en el espacio actual de la disrupción financiera, la era digital y la pandemia originada por la covid-19.

\section{Marco teórico}

La revolución tecnológica, la industria 4.0 o la industria inteligente ${ }^{1}$ constituyen todo el contexto globalizado de la era digital, la cual se sustenta en el uso del internet. Analizando algunos conceptos tecnológicos de forma cronológica, en primera instancia aparece el vocablo bitcoin, que nace en el año 2009 y se define como una moneda virtual independiente y descentralizada ${ }^{2}$ que puede ser utilizada como medio de pago igual que el dinero físico (Finanzas para Todos, 2010, párr. 1). Su mecanismo de funcionamiento se resume de esta manera:

\footnotetext{
${ }^{1}$ La cuarta revolución industrial busca transformar a la empresa en una organización inteligente para conseguir mejores resultados del negocio (CIC, 2019).

${ }^{2}$ Concepto que indica que no está controlada por ningún estado o institución financiera, banco o empresa. (BE, 2010).
} 
[Se descarga] un software en el ordenador o nuestro móvil que hará las veces de un monedero virtual y que generará una dirección bitcoin, que se podrá usar para enviar y recibir dinero de otros usuarios. Además, el envío de bitcoins es instantáneo y toda operación puede ser monitorizada en tiempo real. Las transacciones con esta moneda implican una transferencia de valor entre dos direcciones bitcoin, públicas, aunque anónimas. Para garantizar la seguridad, las transacciones son aseguradas utilizando una serie de criptografías de llaves, ya que cada cuenta dispone de una llave pública y otra privada (Finanzas para Todos, 2010, párr. 4).

Ahora bien, el concepto criptoactivos se define del siguiente modo:

Conjunto de activos digitales y nuevos bienes y servicios basados en la criptografía y la blockchain para su funcionamiento. Representa los valores de los cuales se espera obtener un beneficio económico a corto, mediano o largo plazo (...). Un activo digital es aquello que existe solo de forma binaria y que da ciertos derechos a su poseedor. Ello gracias a que se puede usar, disfrutar y disponer del activo (Garrido, 2019, párr 3).

Este concepto nace con Wei Dai ${ }^{3}$ en el año de 1998, y después de casi 20 años en el mercado virtual, se convirtió en el medio de inversión y pago que ha presentado gran interés por los diversos beneficios que genera, aunque también por los usos ilícitos que los delincuentes le han dado.

En el año 2014, el Grupo de Acción Financiera Internacional ${ }^{4}$ (GAFI) emitió el documento intitulado Monedas virtuales: principales definiciones clave y riesgos potenciales en PLD / CFT ${ }^{5}$, donde se define a la criptomoneda como "moneda virtual convertible descentralizada fundamentada matemáticamente y que está protegida por criptografía, es decir que incorpora los principios de la criptografía para implementar una economía de la información segura, descentralizada y distribuida" (GAFI, 2014, p. 5).

\footnotetext{
${ }^{3}$ Reconocido criptógrafo y miembro de la comunidad cypherpunk, muy conocido por sus aportes al campo de estudio. En el mundo de las criptomonedas, será recordado por situar una propuesta clave precursora de bitcoin justo antes de la creación de Satoshi Nakamoto y por la librería “crypto++" (Bit2me, 2015).

4 Institución intergubernamental creada en 1989 cuyo propósito es desarrollar políticas que ayuden a combatir el blanqueo de capitales y el financiamiento al terrorismo.

${ }^{5}$ Prevención de lavado de dinero / combate de financiamiento al terrorismo.
} 
Por otra parte, el término tecnologías financieras y la palabra fintech, es referida en el diario digital Oro y Finanzas de este modo:

Fintech (...), acrónimo de dos palabras inglesas Financial Technology, es decir tecnología financiera, se ha convertido en el término omnipresente para referirse a cualquier tecnología aplicada a los servicios financieros. Podría definirse, por tanto, como la innovación tecnológica en los servicios financieros siguiendo el patrón de lo que se ha hecho desde el sector de la tecnología con las industrias de los medios de comunicación, turismo y telecomunicaciones en los últimos 15 años (Oro y Finanzas, 2015, párr. 2).

Tomando como referencia que las tecnologías disruptivas se consideran cambios innovadores que modifican las diferentes condiciones de nuestro entorno o modo de vida, entonces las tecnologías financieras se refieren a empresas que utilizan la innovación digital para ofrecer servicios más eficaces y económicos que también se pueden ofrecer a la población que no cuenta con la banca tradicional para efectuar compras y envíos de forma digital en monederos electrónicos (walletes) por medio de teléfonos inteligentes.

Una de las políticas de inclusión financiera del 21 de junio de 2016 fue incrementar el uso de las innovaciones tecnológicas; en consecuencia $-\mathrm{y}$ según los resultados del $15 .^{\circ}$ Estudio sobre los Hábitos de los Usuarios de Internet en México 2018, (AIM, 2019), el número de usuarios de internet en el año 2018 creció $4.3 \%$ en comparación con el año anterior, los cuales usaban principalmente la computadora de escritorio (20\%) y los dispositivos móviles (28\%). A la par de este aumento, sin embargo, también vale acotar que la cantidad de ciberdelitos se ha incrementado, como son los robos de identidad, la falsificación de tarjetas de crédito, el ataque a servidores de empresas, el jaqueo de cuentas bancarias, así como el robo de información confidencial de organismos públicos y privados. De hecho, “en 2017, el cibercrimen costó 600000 millones dólares a la economía mundial, lo que representa el $.08 \%$ del PIB global, según datos de McAfee y el CIIS (Center for Strategic and International Studies)” (Staff, 2019, párr. 3).

En la actualidad, los criptoactivos o monedas virtuales pretenden ser o han sido utilizados por los delincuentes como un nuevo método para el blanqueo de capitales. Esto significa que las tecnologías se han vuelto el modo operante de las actividades económicas, de ahí que la inclusión financiera de los contribuyentes se haya convertido en un objetivo 


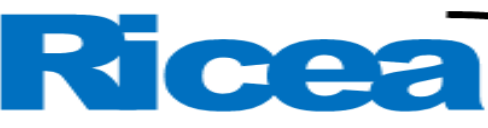

REVISTA IBEROAMERICANA DE CONTADURÍA, ECONOMÍA Y ADMINISTRACIÓN

ISSN $=2007-9907$

trazado por la administración federal 2012-2018 para emitir normativas orientadas a la prevención de esta nueva modalidad criminal.

\section{Empresas fintech}

Las empresas fintech pueden ser concebidas como entidades que ofrecen productos y servicios financieros mediante el uso de tecnologías de la información y la comunicación, como páginas de internet, redes sociales y aplicaciones para celulares, lo cual brindaría un servicio menos costoso que el ofrecido por la banca tradicional (Comisión Nacional para la Protección y Defensa de los Usuarios de Servicios Financieros [Condusef], 2017, párr. 1). En la tabla 1 se observan las verticales más importantes de estas empresas, según la Asociación de Fintech en México: 


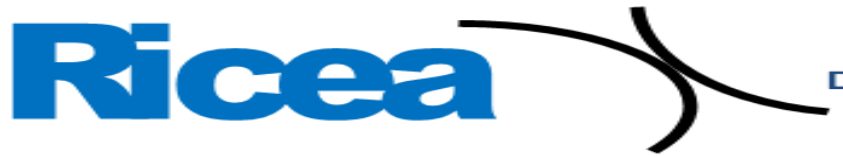

Tabla 1. Diversos servicios de empresas fintech

\begin{tabular}{|c|c|}
\hline Servicios & Particularidad \\
\hline Medios de pago y transferencias & $\begin{array}{l}\text { Plataformas de pago, comercio electrónico y } \\
\text { orientada internacionales. }\end{array}$ \\
\hline $\begin{array}{l}\text { Infraestructura para } \quad \text { servicios } \\
\text { financieros }\end{array}$ & $\begin{array}{l}\text { Evaluación de riesgo, perfiles de clientes y de } \\
\text { riesgo, prevención de fraudes, verificación de } \\
\text { identidades } \mathrm{API}^{6} \text { bancarias, agregadores de } \\
\text { medios de pago, inteligencia de negocios, } \\
\text { ciberseguridad y contratación electrónica. }\end{array}$ \\
\hline Contratación digital de créditos & $\begin{array}{l}\text { Empresas que ofrecen productos de crédito a } \\
\text { través de plataformas electrónicas. }\end{array}$ \\
\hline Soluciones financieras para empresas & $\begin{array}{l}\text { Software para contabilidad e infraestructuras de } \\
\text { facturación y gestión financiera. }\end{array}$ \\
\hline $\begin{array}{l}\text { Finanzas personales y asesorías } \\
\text { financieras }\end{array}$ & $\begin{array}{l}\text { Administración de finanzas personales, } \\
\text { comparadores y distribuidores de productos } \\
\text { financieros, educación financiera, asesores } \\
\text { automatizados y planeación financiera. }\end{array}$ \\
\hline Mercados financieros & $\begin{array}{l}\text { Servicios digitales de intermediación de valores, } \\
\text { instrumentos financieros y divisas. }\end{array}$ \\
\hline Crowdfunding & $\begin{array}{l}\text { Modelo de financiamiento colectivo donde } \\
\text { diferentes personas de diversas partes del mundo } \\
\text { realizan pequeñas o grandes aportaciones } \\
\text { financieras a un determinado proyecto. }\end{array}$ \\
\hline Insurtech & $\begin{array}{l}\text { Tecnología aplicada a la prestación de servicios } \\
\text { en el sector asegurador }\end{array}$ \\
\hline Criptomonedas y blockchain & $\begin{array}{l}\text { Desarrolladores de soluciones basados en } \\
\text { blockchain, intermediarios digitales y mercados } \\
\text { de activos tangibles. }\end{array}$ \\
\hline Entidades financieras disruptivas & $\begin{array}{l}\text { Bancos u otras entidades financieras } 100 \% \\
\text { digitales. }\end{array}$ \\
\hline
\end{tabular}

Fuente: Elaboración propia con base en Fintech México (s. f.)

Los servicios descritos en la tabla 1 representan una manera práctica de realizar las diversas operaciones ofrecidas aunque eliminando el contacto físico entre los prestadores de servicios y los usuarios, situación que puede ser aprovechada por los criminales para, por ejemplo, el lavado de activos.

Las empresas fintech tienen su origen en la década de 1950 con la emisión de las primeras tarjetas de crédito. Luego, en la década de 1960 surgen los primeros cajeros automáticos, y en 1970 se empiezan a popularizar las acciones electrónicas, lo cual se fortaleció en 1990 con el florecimiento de los comercios electrónicos y posteriormente con

${ }^{6}$ Application programming interface. 
la creación de $\mathrm{PayPal}^{7}$ en el año 2002 (Hipodec, 2018, párr. 3). En el caso concreto de México, Mejía (2019) señala lo siguiente:

Las 515 empresas fintech que existen en México, desde 2015 se han levantado capital y deuda por un monto equivalente a 629 millones de dólares. En materia de empleo, el ecosistema Fintech ha creado 3600 empleos directos y beneficia a más de 4.5 millones de usuarios registrados en sus servicios. Del total de las empresas, $80 \%$ se encuentra ya operando: $48 \%$ en etapa de crecimiento y expansión, y 355 están listas para escalar (párr. 3).

Las empresas fintech resultan para los usuarios mas fáciles de operar. Además, el alcance y la rapidez para realizar las diferentes transacciones que se ofrecen resultan más atractivos. Al respecto, Hipodec (2018) refiere:

A diferencia de las instituciones bancarias, las Fintech ofrecen nuevas posibilidades a las personas que no necesitan manejar cuentas complicadas, así como tienen un alcance geográfico más desinteresado; sin embargo, a medida que siguen creciendo, continúan consolidando (y requiriendo) políticas de operación y comportamiento para otorgar mayor seguridad, la cual aún no puede compararse con las grandes corporaciones (párr. 5).

Ante la necesidad de normar el marco operacional de las denominadas empresas fintech, el $1 .^{\circ}$ de marzo de 2018 entró en vigor la ley para regular a las instituciones de tecnología financiera (denominada ley fintech), así como la emisión de las Disposiciones de Carácter General (DCG) aplicables a estas empresas, publicadas en el Diario Oficial de la Federación (DOF) el 10 de septiembre de 2018 y modificadas mediante una resolución con fecha 25 de marzo de 2019, en donde se faculta a la Comisión Nacional Bancaria y de Valores (CNBV) para lo siguiente:

[Emitir DCG] bajo los principios de inclusión e innovación financiera, promoción de la competencia, protección al consumidor, preservación de la estabilidad financiera y neutralidad tecnológica y que dichas disposiciones incluyen requisitos para actuar como institución de tecnología financiera, capitales mínimos, contabilidad, excepciones para recibir recursos en efectivo o efectuar y recibir transferencias nacionales o internacionales (...) a fin de

\footnotetext{
${ }^{7}$ Plataforma de pago asociado a una tarjeta de crédito o débito que se utiliza para las compras en línea con el beneficio de no proporcionar los datos personales al vendedor o proveedor de servicios.
} 
destinar los recursos a los esquemas para la alineación de incentivos de las instituciones de financiamiento colectivo, constancias sobre riesgos, metodologías sobre la asignación del grado de riesgo a los solicitantes de recursos en instituciones de financiamiento colectivo y plan de continuidad de negocios entre otras (CNBV, 2019a, párr. 3).

La Comisión Nacional Bancaria y de Valores refiere que México ocupa el séptimo lugar a nivel mundial en la regulación del sector fintech y señala que existen alrededor de 330 empresas; sin embargo, en un comunicado de fecha $1 .^{\circ}$ de octubre de 2019 , la CNBV manifestó que tras concluir el plazo para solictar autorización para operar como instituciones de tecnología financiera únicamente recibió un total de 85 empresas, de las que 60 solicitaban autorización para operar como instituciones de fondos de pago electrónico y 25 como instituciones de financiamiento colectivo (CNBV, 2019b, párr. 1).

Las principales figuras que contempla la regulación y supervisión de la Secretaría de Hacienda y Crédito Público y la Comisión Nacional Bancaria y de Valores se resumen en la tabla 2: 


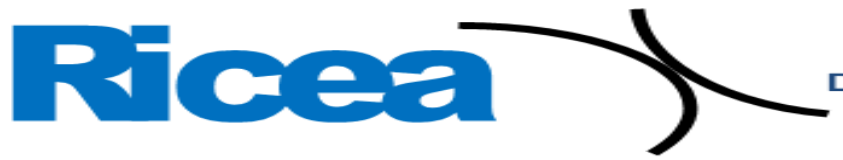

Tabla 2. Instituciones fintech supervisadas por la CNBV

\begin{tabular}{|c|c|}
\hline Institución & Operaciones \\
\hline $\begin{array}{l}\text { Instituciones de Fondos de } \\
\text { Pago Electrónico (IFPE) }\end{array}$ & $\begin{array}{l}\text { - Emisión, admnistración y redención de saldos de } \\
\text { dinero registrados electrónicamente para hacer } \\
\text { pagos o transferencias. } \\
\text { - Pueden realizar operaciones en moneda nacional } \\
\text { y virtuales. } \\
\text { - Pueden actuar como transmisor de dinero. } \\
\text { - Pueden comercializar, emitir o administrar } \\
\text { medios de disposición. }\end{array}$ \\
\hline $\begin{array}{l}\text { Instituciones de Financiamiento } \\
\text { Colectivo (IFC) (Crowfunding } \\
\text { financiero) }\end{array}$ & 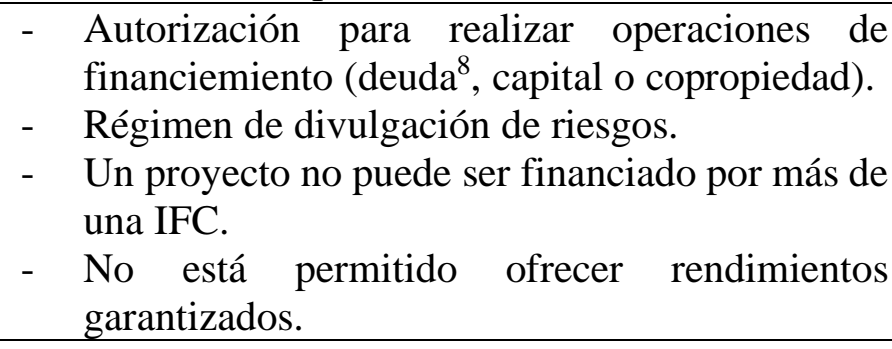 \\
\hline $\begin{array}{l}\text { Activos virtuales } \\
\text { (criptomonedas) }\end{array}$ & $\begin{array}{l}\text { - Las IFPE e IFC podrán operar con los activos } \\
\text { virtuales autorizados por Banxico. } \\
\text { - Banxico definirá las condiciones y restricciones } \\
\text { de las operaciones realizadas con activos } \\
\text { virtuales. } \\
\text { - Cualquier institución que maneje activos } \\
\text { virtuales deberá sujetarse a la regulación } \\
\text { aplicable en materia de PLD. }\end{array}$ \\
\hline
\end{tabular}

Fuente: Elaboración propia con base en CNBV (2018a)

Como se observa, antes de la implementación de la normatividad para operar bajo la figura fintech, estas empresas eran alrededor de 515; sin embargo, únicamente 85 entidades obtuvieron la autorización de la CNBV para realizar los servicios señalados en la tabla 2. Tales autorizaciones son conferidas por el Comité Interinstitucional de Integración, que se encuentra constituido por dos representantes de la Secretaría de Hacienda y Crédito Público, dos del Banco de México y dos de la Comisión Nacional Bancaria y de Valores, con un total de seis miembros y fungiendo como su presidente uno de los funcionarios de la CNBV.

Las Disposiciones de Carácter General emitidas por la CNBV aplicables a las instituciones de tecnología financiera están conformadas por cuatro títulos, diversos capítulos y secciones, así como un listado de anexos tanto para las instituciones de pago electrónico como para las instituciones de financiamiento colectivo (crowfunding financiero). En el título segundo, capítulo I, se describe la información y documentación para solicitar la autorización

\footnotetext{
${ }^{8}$ Las que corresponden a deuda deben usar al menos un buró de crédito (CNBV, 2018a, párr. 7).
} 


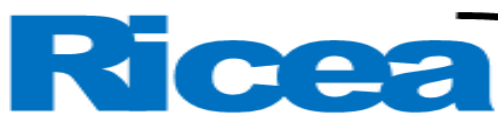

REVISTA IBEROAMERICANA DE CONTADURÍA, ECONOMÍA Y ADMINISTRACIÓN

ISSN $=2007-9907$

para operar como ITF, así como otros requisitos que se debieron cumplir para su operatividad en cuanto a los estatutos sociales, instrumento público, plan de negocios, documentos de políticas en materia de control de riesgos, responsabilidades de las operaciones, procesos operativos de control, de identificación de clientes, de resolución de conflicto de interés y de prevención de fraudes de operaciones con recursos de procedencia ilícita, de contabilidad, valuación de valores y otros instrumentos financieros, así como activos virtuales (Documento completo en disposiciones de carácter general aplicables a las instituciones de tecnología financiera).

\section{Activos virtuales}

La tecnología cuenta con diferentes condiciones para perfeccionar las operaciones realizadas por las empresas prestadoras de los servicios financieros. En la regulación establecida por la CNBV se señala que el Banco de México (Banxico) definirá las condiciones y restricciones para las operaciones efectuadas con activos virtuales. Al respecto, Banxico señala esto:

Los activos virtuales surgieron como una propuesta de esquema alternativo para realizar pagos, en el cual se evita la participación de un tercero confiable en el proceso de una transacción. Sin embargo, por sus características, estos activos han presentado diversos problemas para ser un sustituto de la moneda, pues no cumplen con las características del dinero: depósito de valor, medio de cambio ni unidad de cuenta (Banco de México [Banxico], s. f. párr. 1).

Banxico describe las características generales para diferenciar a la moneda de uso legal o producto financiero y a los activos virtuales, como se describen en la tabla 3: 


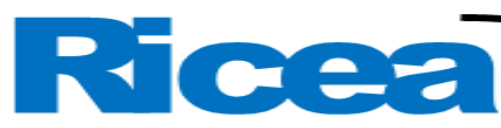

Tabla 3. Características generales del efectivo, transferencia electrónica y activo virtual

\begin{tabular}{|c|c|c|c|}
\hline & Efectivo & Trasferencia electrónica & Activo virtual \\
\hline Posesión & $\begin{array}{l}\text { Billetes } \\
\mathrm{y} \\
\text { monedas }\end{array}$ & $\begin{array}{l}\text { Registros de los saldos de } \\
\text { las cuentas de depósito } \\
\text { que lleve una institución } \\
\text { financiera. }\end{array}$ & $\begin{array}{l}\text { Posesión de una llave } \\
\text { privada que permite iniciar } \\
\text { transferencias dentro del } \\
\text { registro distribuido de } \\
\text { dicho activo. }\end{array}$ \\
\hline Almacenamiento & Físico & $\begin{array}{l}\text { Digital, almacenado en } \\
\text { los registros de la } \\
\text { institución financiera. }\end{array}$ & $\begin{array}{l}\text { Digital, almacenado en los } \\
\text { nodos de la red distribuida } \\
\text { del activo virtual. }\end{array}$ \\
\hline $\begin{array}{l}\text { Método de } \\
\text { intercambio }\end{array}$ & Físico & $\begin{array}{lr}\text { Digital, } & \text { mediante } \\
\text { instrucciones } & \text { de } \\
\text { transferencia a la } \\
\text { institución financiera que } \\
\text { lleve a su cargo las } \\
\text { cuentas de depósito. }\end{array}$ & $\begin{array}{l}\text { Digital, mediante un } \\
\text { mensaje que se envía a la } \\
\text { red del activo virtual a } \\
\text { través de internet. }\end{array}$ \\
\hline Doble gasto & $\begin{array}{l}\text { Se evita mediante } \\
\text { el intercambio de } \\
\text { algo tangible } \\
\text { (billetes ry } \\
\text { monedas). }\end{array}$ & $\begin{array}{l}\text { Se evita a traves de la } \\
\text { validación de los saldos } \\
\text { en las cuentas de depósito } \\
\text { que lleve a su cargo una } \\
\text { institución financiera. }\end{array}$ & $\begin{array}{l}\text { Se evita mediante la } \\
\text { validación de tenencia a } \\
\text { partir de la revisión del } \\
\text { registro distribuido que } \\
\text { contiene el histórico de las } \\
\text { transacciones, así como } \\
\text { mediante el proceso de } \\
\text { minado. }\end{array}$ \\
\hline Falsificación & \begin{tabular}{l}
\multicolumn{3}{l}{ Se evita mediante } \\
elementos de \\
seguridad físicos \\
que evitan la \\
duplicación de \\
billetes.
\end{tabular} & & \\
\hline Respaldo & Banco Central & $\begin{array}{l}\text { Institución financiera que } \\
\text { lleve a su cargo las } \\
\text { cuentas de depósito y los } \\
\text { registros de los saldos. }\end{array}$ & $\begin{array}{l}\text { No hay un respaldo; está } \\
\text { basado en la confianza de } \\
\text { sus usuarios en la red del } \\
\text { activo virtual. }\end{array}$ \\
\hline
\end{tabular}

Fuente: Banxico (s. f.)

\section{Criptoactivos}

Las criptomonedas son activos virtuales fáciles de utiliar, ya que se pueden aplicar para realizar pagos o cualquier otra operación comercial. En la actualidad - y según la información referida por CoinMarketCap (2020)— hasta el 22 de abril de 2020 existen 5392 criptomonedas con un capital de mercado de \$205 250839 mmdp. Entre los criptoactivos más utilizados la tabla 4 destaca los siguientes: 
Tabla 4. Criptoactivos más utilizados

\begin{tabular}{|l|l|}
\hline Bitcoin & $\begin{array}{l}\text { El más antiguo y líder en el mercado, de gran aceptación y confianza para } \\
\text { la comunidad que lo utiliza. Ofrece eficacia, transparencia, seguridad, } \\
\text { anonimato y comisiones aceptables tanto para invertir como para ser } \\
\text { utilizado como medio de pago. }\end{array}$ \\
\hline Ethereum & $\begin{array}{l}\text { Criptodivisa rentable para invertir con plataforma que ofrece excelentes } \\
\text { propuestas a los usuarios, permite la creación de contratos inteligentes. }\end{array}$ \\
\hline Ripple & $\begin{array}{l}\text { Utiliza un proyecto de software libre y se encuentra en el ranking de las } \\
\text { criptomonedas más rentables en el mercado, y las más usadas para realizar } \\
\text { transacciones financieras a nivel global, en tiempo real, de forma } \\
\text { descentralizada y segura. }\end{array}$ \\
\hline Litecoin & $\begin{array}{l}\text { Criptomoneda descentralizada con mayor rendimiento. Permite enviar y } \\
\text { recibir dinero sin importar la distancia; posee ventajas y beneficios para } \\
\text { usurarios e inversionistas. }\end{array}$ \\
\hline Bitcoin cash & $\begin{array}{l}\text { Criptomoneda sólida, que cumple con la promesa original del bitcoin, es } \\
\text { decir, "dinero electrónico par a par". Los usuarios y comerciantes son } \\
\text { beneficiados con tasas bajas y confirmaciones confiables. De adopción } \\
\text { global, innovación sin necesidad de permisos y desarrollo descentralizado. }\end{array}$ \\
\hline Golem & $\begin{array}{l}\text { El proyecto pretende ser una alternativa para los proveedores centralizados } \\
\text { de servicios en la nube, con su precio más bajo y código abierto para la } \\
\text { comunidad de desarrolladores. Las transacciones entre los participantes se } \\
\text { consideran seguras porque los cálculos se llevan a cabo en el sandbox. }\end{array}$ \\
\hline Agrocoin & $\begin{array}{l}\text { Criptomoneda para invertir en el campo mexicano; creado por una } \\
\text { empresa que produce y comercializa chile habanero, ofrece rendimientos } \\
\text { anuales. }\end{array}$ \\
$\begin{array}{l}\text { Criptomoneda estable que se encuentra respaldada por dólares } \\
\text { americanos. Sus usuarios poseen completa garantía de sus activos y tienen } \\
\text { el derecho de cambiar los tokens por dinero fiduciario en dicha moneda. }\end{array}$ \\
\hline Fuen
\end{tabular}

Fuente: Elaboración propia con base en Molina (2019) y Cash (2020)

Los criptoactivos descritos en la tabla 4 se pueden clasificar de este modo:

- Criptomonedas para pago, como es el caso del bitcoin, riplle y bitcoin cash.

- Activos virtuales como un medio de productos o servicios como el Ethereum o Golem.

- Activos virtuales con subyacentes o adyacentes ${ }^{9}$, como es el caso de agrocoin y trueusd.

El uso de los criptoactivos en diversas operaciones presenta ventajas y desventajas, algunas de éstas se explican en la tabla 5.

\footnotetext{
${ }^{9}$ Que son respaldados por algún activo.
} 
Tabla 5. Ventajas y desventajas de los criptoactivos

\begin{tabular}{|l|l|}
\hline Ventajas & Desventajas \\
\hline $\begin{array}{l}\text { Se puede tener un monedero sin necesidad } \\
\text { de disponer de acceso a los servicios } \\
\text { bancarios. }\end{array}$ & Las transacciones son irreversibles. \\
\hline $\begin{array}{l}\text { Los pagos se realizan de forma casi } \\
\text { instantánea, no importa en qué parte del } \\
\text { mundo estén el emisor y el receptor. }\end{array}$ & $\begin{array}{l}\text { Alta volatilidad y cambios drásticos en su } \\
\text { cotización. }\end{array}$ \\
\hline $\begin{array}{l}\text { Las operaciones son directas, los usuarios } \\
\text { tienen el control total sobre su dinero. }\end{array}$ & $\begin{array}{l}\text { Por su fase de desarrollo no existen } \\
\text { aplicaciones necesarias para utilizar el } \\
\text { criptoactivo de forma fácil y sencilla. }\end{array}$ \\
\hline $\begin{array}{l}\text { Las comisiones al realizar transferencias de } \\
\text { valor son muy bajas; los mismos usuarios } \\
\text { pueden decidir qué tasa quieren pagar para } \\
\text { dar velocidad a la operación. }\end{array}$ & \\
\hline $\begin{array}{l}\text { Utilizan un sistema basado en tecnología } \\
\text { blockchain, un servicio gratuito y abierto } \\
\text { para todo el mundo. }\end{array}$ & \\
\hline
\end{tabular}

Fuente: Elaboración propia con base en Chacón (s. f.)

\section{El delito de lavado de activos}

El delito de lavado de activos, tipificado en el artículo 400bis del Código Penal Federal, referido como "Operaciones con recursos de procedencia ilícita”, tradicionalmente llamado lavado de dinero, representa en la actualidad aproximadamente $5 \%$ del producto interno bruto (PIB) mundial. Sobre este delito, México ha estado en constante lucha para prevenirlo, siguiendo las recomendaciones hechas por el GAFI como resultado de las evaluaciones mutuas concretadas (la primera en el año 2008 y la última en el año 2018). En ellas se señala, principalmente, que el país cuenta con un régimen de PLD/CFT que ha tenido mejoras significativas en algunas áreas. En dichas evaluaciones también se indica que las autoridades y el sector financiero tienen una buena comprensión sobre los riesgos y amenazas que implica dicho régimen. Además, se apuntan las fortalezas y debilidades de la Unidad de Inteligencia Financiera, así como de la Procuraduría General de la República en torno a la importancia de dar seguimiento al decomiso de los activos provenientes del crimen o la identificación y diligencia del beneficiario real de los recursos. (Informe completo de evaluación mutua en Informe de Evaluación Mutua 2018). En síntesis, el GAFI reconoce la innovación financiera, así como los productos y servicios de las "monedas virtuales (MV) 
(VCPPS $^{10}$ por sus siglas en inglés) que presentan riesgos de lavado de activos y financiamiento terrorista (LA/FT) y otros riesgos de delitos que deben ser identificados y mitigados" (GAFI, 2015, párr. 1).

Las DCG refieren que cualquiera de las empresas fintech constituidas como instituciones de fondos de pago electrónico o instituciones de financiamiento colectivo que operen con activos virtuales autorizados por Banxico deberán sujetarse a la regulación aplicable en materia de PLD. La CNBV (2018b, párr. 7) refiere las medid

as y procedmientos mínimos de control que las instituciones de tecnología financiera (ITF) deben cumplir con las siguientes obligaciones en materia de PLD / FT:

- Manual de PLD/FT.

- Metodología de evaluación de riesgos.

- Estructuras internas (oficial de cumplimiento, oficial de cumplimiento interino y comité de comunicación y control).

- Sistemas automatizados.

- Reportes.

- Informe de auditoría.

El manual de PLD/FT deberá integrar una política de identificación del cliente, la cual tiene que señalar que las ITF están obligadas a integrar expedientes de cada uno de sus clientes previo a la celebración de contratos, prestación de servicios y realización de las operaciones, así como solicitar a estos la geolocalización del dispositivo electrónico móvil desde el que se abra la cuenta o se celebre el contrato. Asimismo, deberán contar con un sistema automatizado que desarrolle funciones como conservar, actualizar datos y documentos, validar su autenticidad proporcionados de forma remota, generar y transmitir los diferentes reportes a la secretaría por conducto de la CNBV, garantizar la trazabilidad y certeza del origen y destino de los activos virtuales con los que operen, clasificar los tipos de operaciones, productos o servicios, monitorear y agrupar en una base consolidada las diferentes operaciones de un mismo cliente e incluir una metodología basada en riesgos, entre otras. Asimismo, las ITF deberán cumplir con la normatividad aplicable sobre el envío de reportes, entre otros el de operaciones con activos virtuales.

\footnotetext{
${ }^{10}$ Virtual Currencies Key Definitions and Potencial AML/CFT Risks.
} 


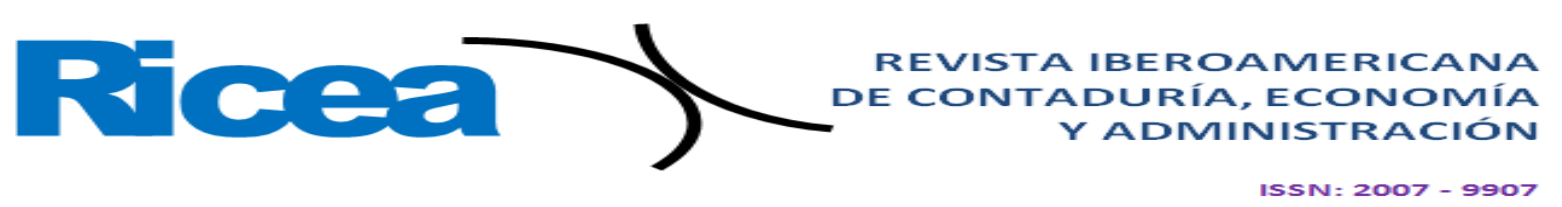

\section{Resultados}

¿Cuáles son los retos y oportunidades que enfrentan las fintech y el uso de las tecnologías financieras? Los resultados se presentan en orden cronológico. Bécares (2017), un año antes de la implementación de la regulación en México indica que, en un análisis realizado a funcionarios de Aceleradora Startupbootcamp, Prestadero y Afluenta Eduardo Morelos (Párr.4) menciona que"las Fintech están cobrando mayor protagonismo buscando dar solución a través de la tecnología a problemas donde la industria tradicional no ha sabido llegar”. Asimismo, Gerardo Obregón (Párr.5), director general de Prestadero, señala que "aunque hay un sector de la población que aún desconfía en las nuevas opciones de los servicios financieros, esta es una barrera que desaparecerá con las nuevas generaciones. Es un proceso paulatino, pero de mucho esfuerzo de nosotros ara inspirar confianza". Alejandro Cosentino (Párr. 6), fundador y CEO de Afluenta, explica que "en el panorama mexicano más de $50 \%$ de las personas tienen un teléfono inteligente y lo consideran como primer dispositivo en cuanto a las preferencias de uso en general; ya no quieren ir a una sucursal bancaria".

Entre los retos, Demetrio Strimpópulos (Párr.6), director de BanRegioLABS, considera que "el desarrollo de emprendimiento Fintech se fortalecerá si creamos un ambiente de colaboración adecuado entre los diferentes actores: banca, emprendedores, fondos de capital y entes reguladores". Obregón (Párr.7) opina que "los principales retos son la regulación y la educación financiera", además señala "que las Fintech y las nuevas tecnologías están incrementando la competencia y aumentando las opciones para los consumidores". Alejandro Cosentino (Párr. 8) - de Afluenta - cree que, "explicar de manera clara y simple a los usuarios mexicanos como las Fintech mejoran las condiciones de los préstamos entre personas, así como brindar información sobre la transparencia de las operaciones que se realizan respetando el marco regulatorio local". Cosentino (Párr.10) menciona también "en la era digital, la tendencia natural es hacia ese tipo de servicios y que se optimizan recursos, se favorece la inclusión financiera y la experiencia de los usuarios".

El 1. ${ }^{\circ}$ de marzo de 2018 entra en vigor la ley fintech, y la CNBV reporta que solo 85 de las 303 empresas de esa naturaleza obtuvieron el permiso para operar como ITF. Cobis (2019) refiere que nuevas generaciones como los millennials marcan la pauta a las fintech, pues buscan desarrollar procesos más sencillos que los de la banca tradicional. (Párr.6) Sin embargo, estas también quieren llegar a más personas, en el mismo documento Adrián 
Fernández de Mendoza, (Párr.7) director general de Creditea México menciona: "Se debe tener en cuenta al segmento de la gente que nació digital, y por eso es complicado operar entre la rigidez y la informalidad. Se debe actuar como banca tradicional para llegar a la gente mayor, pero al mismo tiempo proyectar una imagen innovadora y vanguardista".

En cuanto a los activos virtuales, en Deloitte (2019) Carlos Orta — socio de Risk Advisory en Deloitte México - explica que Banxico señala en su circular 4/2019 los riesgos que actualmente existen al operar con estos, a los que califica como volátiles, costosos y difícilmente escalables (Párr.6); Orta subraya los peligros que enfrentan los tenedores de estos activos, principalmente por la complejidad de la tecnología que los soporta y el desconocimiento que la mayoría tiene sobre su funcionalidad y sobre los problemas que se puedan presentar.(Párr. 3) Aunado a esto, se debe tener en cuenta el riesgo subyacente en materia de lavado de dinero y financiamiento del terrorismo, ya que los activos pueden ser fácilmente transferidos a otros países, de ahí que sean necesarias medidas de prevención uniformes a nivel global. (Párr. 6)

En entrevista realizada por Gutiérrez (2019) en el Economista, Pablo González — cofundador del exchanche de criptomonedas Bitso - apuntó que si bien las criptomonedas han llegado para quedarse, todavía hay una serie de retos que se tienen que enfrentar para tener una mayor adopción; en el caso de México, por ejemplo, aunque ya existe una ley para normar este tipo de activos, la tecnología puede avanzar más rápido que la regulación. González también detalló que desde que Bitso inició operaciones en México, la adopción ha sido gradual, pero constante.

Estimamos que actualmente el tamaño del mercado en México es de 800000 usuarios utilizando criptomonedas (...). El mercado es grande, pero nos gustaría que llegara a un mayor porcentaje de la población. Todavía hay gente que desconoce los beneficios de traer a sus vidas la tecnología y queremos darles la vuelta a estos estigmas. (Párr. 4)

En términos generales, la evolución ha pasado de una trasformación tecnológica a tener casos de usos reales, como las remesas o pagos. Sobre la ley fintech, González apuntó que es un gran precedente para establecer reglas claras del juego, tanto para reguladores como para consumidores y participantes de la industria. En el mundo de la innovación es normal que las tecnologías avancen más rápido que la regulación, por lo que se requiere una amplia apertura para comprender los beneficios de estas nuevas tecnologías. En cuanto a las 


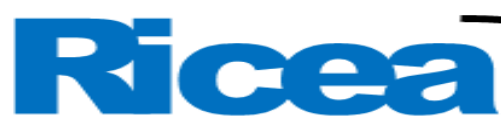

REVISTA IBEROAMERICANA DE CONTADURÍA, ECONOMÍA Y ADMINISTRACIÓN

ISSN $=2007-9907$

criptomonedas, mencionó que los que trabajamos en el sector no somos los únicos que creemos que las criptomonedas llegaron para quedarse. Un reporte que publicó Greenwich Associates en septiembre de 2019 menciona que $70 \%$ de los ejecutivos del sector financiero también cree que las criptomonedas llegaron para quedarse, de ahí que haya empezado el desarrollo de esta tecnología. En la sierra de Puebla hay mexicanos que cobran remesas de sus familiares en Estados Unidos por medio del bitcoin, a pesar de que no entiendan qué es o cómo funciona esta tecnología. Lo importante para ellos es recibir el dinero y confiar en un servicio más transparente, rápido y conveniente. En pocas palabras, "su uso ya está mejorando la vida de un gran número de familias en nuestro país" (Párr.10).

El pasado 20 de abril de 2020, en entrevista realizada al LFB Daniel Alberto Ortiz de Montellano Velázquez, manager, Forensic, de la firma KPMG en México indica:

¿En la actualidad cuál es el panorama de las empresas Fintech?

En general, todas las empresas están teniendo un auge tecnológico; no solo las de servicios bancarios, con la disrupción de los servicios financieros y considerando que una gran parte de la población es generación de los millennials. Estas empresas están creciendo, se está invirtiendo mucho en ellas y se están saturando.

¿Las operaciones con activos virtuales en la situación actual representan un riesgo para los usuarios de estos?

En general, cualquier inversión es riesgosa. Desde el principio de las inversiones, “a mayor inversión mayor rendimiento", sin embargo, las operaciones con activos virtuales no son riesgosas por la cadena blockchain (cadena de bloques) con una transacción $100 \%$ rastreable; el riesgo que se tiene es por la volatilidad que tienen.

¿Qué ventajas y desventajas considera usted que existen al operar con activos virtuales?

Una de las ventajas es que puedes maximizar los rendimientos porque la inversión es $100 \%$ trazable; las desventajas, entre otras, son que es un tema desconocido para muchos, por lo que pueden ser víctimas de fraude.

¿En qué situación se encuentran las empresas Fintech establecidas en México y con permiso para operar emitid por la CNBV en relación con otras Fintech internacionales?

La ley en México fue muy limitativa al nada más permitir ciertas actividades. Fue un buen ejercicio. Sin embargo, nos deja en desventaja, ya que cualquier persona se puede ir al otro lado del mundo a establecer una Fintech y operar con activos virtuales en México sin 
tener que estar físicamente en México. Muchos países lo han entendido y no han querido establecer regulación. Por ejemplo, en Hong Kong no hay regulación para Fintech; lo que existe es regulación para bancos virtuales que constituyen una parte de la industria Fintech un poco más avanzado.

¿Cuáles son los riesgos que tienen las Fintech y la operación con activos virtuales?

Los riesgos van enfocados a delitos de ciberseguridad en general, delitos financieros, entre ellos el fraude y lavado de activos, ya que se está eliminando el contacto $100 \%$ cara a cara con el cliente y esto siempre genera un riesgo, por lo que debe tenerse una buena infraestructura de seguridad informática como el Firewall, etc., puede ser muy vulnerable.

¿Como podría darse el delito de lavado de activos en las Fintech?

Por ejemplo, en el Crowfunding se puede pedir que estés financiando un proyecto que al final no es real o que no se vayan a utilizar los recursos para los fines del proyecto, o el financiamiento con recursos ilícitos de " $\mathrm{x}$ " proyecto que al final no es real, en activos virtuales existe todo mercado negro donde se pueden comprar desde pornografía, pasaportes e identidades falsas.

¿Cuál sería la estrategia para poder aplicar un conocimiento debido del cliente?

Utilizar tecnología: desde el reconocimiento facial, biométricos, pruebas de identidad, entre otras. En la medida en que se utilice la tecnología para conocer al cliente y obtener su consentimiento para integrar los datos antes mencionados, además de un monitoreo en tiempo real, herramientas predictivas, no tanto analíticas, te dan un buen conocimiento del cliente.

¿Cómo impacta la crisis de la pandemia actual en este tipo de operaciones?

Impacta para bien, porque al final con la pandemia se elimina el contacto cara a cara, y esto precisamente es una de las bondades de estas empresas. Se prevé que las compañías no solo Fintech sino toda la disrupción tecnológica crezcan en la crisis del covid-19.

¿Qué tipo de programa PLD/CFT implementaría en este tipo de empresas?

Todo programa de PLD debe tener cuatro componentes: políticas, sistemas, entrenamiento y auditoría en este tipo de empresas deben estar enfocados. Es decir, las políticas deben ser muy tecnológicas con sistemas robustos, la auditoría enfocada y el entrenamiento debe ser dirigido hacia los riesgos a los que están expuestas.

En cuanto al tema actual de la pandemia, KPMG (2020) —en el boletín KPMG tendencias - refiere que en medio de la crisis por la covid-19 y por el flujo de información asociado a la pandemia se pueden presentar los siguientes delitos cibernéticos: 


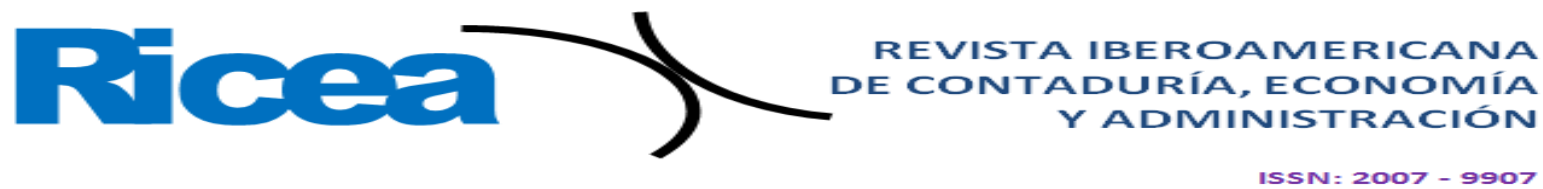

Tabla 6. Modelos de fraudes tecnológicos

\begin{tabular}{|c|c|}
\hline Delito & ¿Cómo se ejecuta? \\
\hline $\begin{array}{l}\text { Phishing } \\
\text { (suplantación } \\
\text { de identidad) }\end{array}$ & $\begin{array}{l}\text { El objetivo es llegar a las víctimas a través de los correos electrónicos con } \\
\text { anexos maliciosos, enlaces o actualizaciones sobre covid-19. Una vez } \\
\text { abiertos, dichos vínculos infectan la computadora o dispositivo móvil con } \\
\text { algún programa maligno (malwere) o exponen datos personales } \\
\text { confidenciales que se transmiten al ciberdelincuente. }\end{array}$ \\
\hline $\begin{array}{l}\text { Sitios webs } \\
\text { fraudulentos } \\
\text { relacionados } \\
\text { con covid-19 }\end{array}$ & $\begin{array}{l}\text { Múltiples dominios de internet han sido registrados con el nombre de } \\
\text { covid. Estos parecen ser auténticos y creados por organizaciones } \\
\text { reconocidas, sin embargo, contienen malware diseñado para infectar las } \\
\text { computadoras u otros dispositivos móviles. }\end{array}$ \\
\hline $\begin{array}{l}\text { Riesgos } \\
\text { relacionados } \\
\text { con el correo } \\
\text { empresarial }\end{array}$ & $\begin{array}{l}\text { Mediante el uso de correos electrónicos disfrazados de actualizaciones de } \\
\text { covid-19, los estafadores intentan engañar a los colaboradores de las } \\
\text { empresas para que entreguen sus credenciales, y después solicitan que } \\
\text { inicien sesión en un falso portal covid-19 de la organización. Una vez que } \\
\text { la persona ingresa con sus credenciales, el defraudador obtiene acceso } \\
\text { ilimitado a las cuentas empresariales y a la red de la compañía. }\end{array}$ \\
\hline $\begin{array}{l}\text { Ataques de } \\
\text { ransomware } \\
\text { (secuestro de } \\
\text { datos) }\end{array}$ & $\begin{array}{l}\text { El primer paso que realizan los estafadores es comprometer la seguridad } \\
\text { de los servidores críticos y las terminales conectadas para después } \\
\text { encriptarlas. El ataque bloquea el sistema operativo y los archivos del } \\
\text { usuario final y no se pueden accesar hasta que se pague un rescate al } \\
\text { atacante, quien suele demandar el pago en bitcoins. A medida que el acceso } \\
\text { remoto a las computadoras se convierta en la norma "para trabajar en } \\
\text { casa", se espera que un incremento de estos ataques paralice la } \\
\text { infraestructura de las tecnologías de la información de las organizaciones } \\
\text { hasta que los delincuentes logren cobrar los rescates. }\end{array}$ \\
\hline $\begin{array}{l}\text { Otras estafas } \\
\text { mediante } \\
\text { aplicaciones } \\
\text { móviles }\end{array}$ & $\begin{array}{l}\text { Los defraudadores están desarrollando o manipulando aplicaciones de } \\
\text { telefonía móvil que aparentan rastrear la propagación de la covid-19. Sin } \\
\text { embargo, una vez instalada la aplicación se infecta el dispositivo con } \\
\text { malwere, que se puede utilizar para obtener información personal, datos } \\
\text { confidenciales o información de las cuentas o tarjetas bancarias. }\end{array}$ \\
\hline Otras estafas & $\begin{array}{l}\text { Solicitud de donaciones para entidades que no existen y que aparentemente } \\
\text { están ayudando a comunidades afectadas por la covid-19 o bien compañías } \\
\text { que están desarrollando vacunas para su prevención. }\end{array}$ \\
\hline
\end{tabular}

Fuente: Elaboración propia con base en KPMG (2020) 


\section{Discusión}

Las empresas fintech, los activos virtuales, los criptoactivos y los ciberdelitos son conceptos que emanan de la innovación tecnológica y la disrupción financiera, y cobran auge en la cuarta revolución industrial, de ahí que diversos expertos hayan analizado los diferentes retos y oportunidades que enfrentan las fintech para su operación como ITF. Entre las oportunidades se puede destacar que estas buscan alcanzar y mejorar el sistema de la banca tradicional llegando a más sectores de la población, con servicios financieros más sencillos, rápidos, eficaces y económicos que se brindan a través de dispositivos móviles. Las fintech, además, necesitan inversión tanto de recursos financieros como de capital humano capacitado para su desarrollo (esto último se puede cubrir con la participación de los millennials o generación digital, multipantalla e hiperconectados).

Entre los retos, se destaca la adecuada colaboración entre los actores (banca, emprendedores, fondos de capital y autoridad reguladora) con el objetivo de optimizar recursos, favorecer la inclusión financiera, crear confianza y seguridad, y la experiencia de los usuarios. Aunado a esto, es importante resaltar que las fintech también deben actuar como banca tradicional para llegar a la gente mayor, aunque sin descuidar la imagen innovadora y vanguardista.

Como se ha explicado a lo largo de estas páginas, las operaciones con activos virtuales son una realidad, pues más de 800000 usuarios operan con estas monedas (las cuales en la actualidad son aproximadamente 5392). Sin embargo, también se debe hacer hincapié en que las empresas y las personas en general deben reforzar sus sistemas de ciberseguridad. Además, las entidades deben conseguir el permiso para operar como ITF con el fin de salvaguardar la integridad de los consumidores y evitar la consecución de los delitos cibernéticos.

Asimismo, para el adecuado uso de las tecnologías financieras y la debida identificación del cliente se deben aplicar procesos electrónicos, como reconocimiento facial, pruebas de identidad o datos biométricos. Estas técnicas aún son desconocidas por algunos sectores de la población, por lo que se deben realizar con la autorización y consentimiento de los clientes y usuarios.

En relación con la reciente regulación para las ITF, se ha disertado que fue muy limitativa, pues únicamente permite la realización de ciertas actividades. Además, el uso de 
las tecnologías avanza más rápido que la reglamentación, lo que puede generar que estas empresas seas constituidas en otros países, pero operar en México sin tener presencia física.

Con la actual pandemia de la covid-19 los defraudadores suelen emplear medios tecnológicos para cometer ciberdelitos (p. ej., fraudes financieros, estafas, secuestro de datos, entre otros), por lo que los usuarios deben estar atentos a las medidas de prevención que difunden diferentes organizaciones y autoridades. Esto lógicamente representa un gran reto debido a la cantidad y flujo de información que circula en las redes, así como al desconocimiento que se tiene de estas, realidad que aumenta con la realización de operaciones y actividades económicas que se desarrollan en la plataforma de internet en el denominado "home office".

\section{Conclusiones}

En el actual contexto generado por la covid-19, los millennials y el uso masivo de los teléfonos inteligentes han generado la necesidad y el reto de usar los activos virtuales, los criptoactivos y las tecnologías financieras. Esto, por supuesto, ha favorecido la inclusión financiera de los consumidores y ha provocado la disminución en el uso del efectivo para concretar operaciones mercantiles, lo cual, sin embargo, también ha traído como consecuencia el aumento de ilícitos en la economía mexicana.

En este complejo contexto, las empresas constituidas como fintech realizan un gran aporte a la economía mexicana tanto en materia de empleo como de servicios prestados. Por eso, las entidades que no obtuvieron el permiso para operar como ITF tienen el reto de cumplir con los requisitos solicitados por la autoridad, pues de ese modo pueden colaborar con la banca, los emprendedores y los entes reguladores en beneficio de los consumidores, clientes y usuarios.

En medio de esta pandemia, y considerando que México ocupa el segundo lugar en cuanto a ciberataques, se requiere que las empresas fomenten entre los usuarios una cultura de ciberseguridad en sus diferentes equipos tecnológicos con el objetivo de prevenir la consecución de los delitos virtuales, como el robo de identidad (phishing) y el lavado de activos. Considerando la mayoría de las actividades económicas actualmente se realizan a través de los medios electrónicos, se plantea como futura línea de investigación la operación, seguridad y vulnerabilidad del uso de las tecnologías financieras, activos virtuales y empresas fintech en las actividades económicas, así como los retos que esto implica. 


\section{Referencias}

Asociación de Internet MX. [AIM] (2019). $15^{\circ}$ Estudio sobre los hábitos de los Usuarios de Internet en México 2018. Recuperado el 20 de abril de 2020, de https://irpcdn.multiscreensite.com/81280eda/files/uploaded/15\%2BEstudio\%2Bsobre\%2Blos $\% 2 \mathrm{BHa} \_$bitos\%2Bde\%2Blos\%2BUsuarios\%2Bde\%2BInternet\%2Ben\%2BMe_xic o\%2B2019\%2Bversio_n\%2Bpu_blica.pdf

Banco de España [BE], (2010). Finanzas para todos. Recuperado el 24 de 03 de 2020, de https://www.finanzasparatodos.es/es/secciones/actualidad/bitcoin.html

Banco de México [Banxico] (s. f.). Sobre los activos virtuales, los riesgos relevantes y el posicionamiento del Banco de México. Recuperado de https://www.banxico.org.mx/sistemas-de-pago/sobre-activos-virtuales-rie.html

Bécares, B. (2017). Fintech en México: un análisis a sus oportunidades y a los retos que debe superar. Recuperado de https://www.siliconweek.com/e-enterprise/startup/fintech-mexico-analisis-oportunidades-los-retos-superar-80108

Bit2me (2015). Quién es Wei Dai. Recuperado de https://academy.bit2me.com/quien-es-weidai/

Cash, B. (2020). BitcoinCash. Recuperado de https://www.bitcoincash.org/es/

Chacón, S. (s. f.). Ventajas y desventajas de las criptomonedas. Recuperado de https://dineroytrabajo.com/ventajas-y-desventajas-de-las-criptomonedas/

Consulting Informático [CIC] (2019). Industría 4.0: revolución industrial. Recuperado de https://www.cic.es/industria-40-revolucion-industrial/

Cobis, B. (2019). Los retos que enfrentan las Fintech para convertise en un motor económico formal en Latinoamérica. Recuperado de https://blog.cobiscorp.com/fintech-retoslatinoamerica

CoinMarketCap (2020). CoinMarketCap. Recuperado de https://coinmarketcap.com/

Comisión Nacional Bancaria y de Valores [CNBV] (2018a). El sector fintech y su regulación en México. Recuperado de https://www.gob.mx/shcp/articulos/el-sector-fintech-ysu-regulacion-en-mexico

Comisión Naciona Bancaria y de Valores [CNBV] (2018b). Acciones y programas. Prevención de lavado de dinero y financiamiento al terrorismo (PLD/FT). Recuperado de https://www.gob.mx/cnbv/acciones-y-programas/instituciones-detecnologia-financiera

\section{(c)}


Comisión Naciona Bancaria y de Valores [CNBV] (2019a). Disposiciones de Carácter General aplicables a las Instituciones de Tecnología Financiera. Recuperado de https://www.cnbv.gob.mx/Normatividad/Disposiciones\%20de\%20car\%C3\%A1cter $\% 20$ general\%20aplicables\%20a\%20las\%20instituciones\%20de\%20tecnolog\%C3\% ADa\%20financiera.pdf

Comisión Naciona Bancaria y de Valores [CNBV] (2019b). CNBC recibió 85 solicitudes de autorización para operar como institución de tecnología financiera. Recuperado de https://www.gob.mx/cnbv/artículos/cnbv-recibio-85-solicitudes-de-autorización-

para-operar-como-institución-de-tecnologia-financiera

Comisión Nacional para la Protección y Defensa de los Usuarios de Servicios Financieros (Condusef) (2017). Educación financiera. Recuperado de https://www.condusef.gob.mx/Revista/PDF-s/2017/203/buro.pdf

Deloitte. (2019). Activos virtuales. Recuperado el 14 de mayo de 2020, de https://www2.deloitte.com/mx/es/pages/dnoticias/articles/activos-virtualesbanxico.html

Finanzas para Todos (2010). Bitcoin: origen, funcionalidades y riesgos de la moneda virtual. Recuperado de https://www.finanzasparatodos.es/es/secciones/actualidad/bitcoin.html

Fintech México (s. f.). Qué es fintech. Recuperado de https://www.fintechmexico.org/qu-esfintech

Garrido, J. M. (21 de octubre de 2019). EGA Futura Nube. Recuperado de https://www.egafutura.com/glosario/criptoactivos

Grupo de Acción Financiera Internacional [GAFI] (2014). Monedas virtuales. Definiciones clave $y$ riesgos potenciales de LA/FT. Recuperado de www.sib.gob.do/pdf/GAFI_PARA_MONEDA_VIRTUAL.pdf

Grupo de Acción Financiera Internacional [GAFI] (2015). Directrices para enfoque basado en riesgo. Monedas virtuales. Recuperado de https://www.fatfgafi.org/media/fatf/documents/Directrices-para-enfoque-basada-en-riesgoMonedas-virtuales.pdf

Gutiérrez , F. (5 de febrero de 2019). En México hay 800000 usuarios de activos virtuales, Bitso. El Economista. $\quad$ Recuperado de 
https://www.eleconomista.com.mx/sectorfinanciero/En-Mexico-hay-800000usuarios-de-activos-virtuales-segun-datos-de-Bitso-20190204-0093.html

Hayes, S KPMG. (12 de mayo de 2020). 6 fraudes cibernéticos que enfrentan las empresas. Recuperado de https://home.kpmg/mx/es/home/tendencias/2020/05/6-fraudesciberneticos-que-enfrentan-las-empresas.html

Hipodec (2018). La historia de las fintech. Recuperado de https://hipodec.up.edu.mx/blog/lahistoria-de-las-fintech

Mejía , G. (2019). El sector fintech en México ya tiene el tamaño de un banco, pero... Entrepreneur. Recuperado de https://www.entrepreneur.com/article/335486

Molina, J. (2019). Cuántas criptomonedas existen en la actualidad. Recuperado de https://criptotendencia.com/2019/10/09/cuantas-criptomonedas-existen-en-laactualidad/

Oro y Finanzas (2015). Qué es y qué significa fintech. Recuperado de https://www.oroyfinanzas.com/2015/03/que-significa-fintech/

Staff, F. (5 de mayo de 2019). Cibercrimen afecta a uno de cada cuatro mexicanos, según aseguradoras. Forbes México. Recuperado de https://www.forbes.com.mx/cibercrimen-afecta-a-uno-de-cada-cuatro-mexicanossegun-aseguradoras/ 